\title{
Major Depression, Antidepressant Medication and the Risk of Obesity
}

(Running Head: Major Depression and Obesity)

Scott B. Patten*

Jeanne V.A. Williams**

Dina H. Lavorato**

Lauren Brown***

Lindsay McLaren**

Michael Eliasziw**

* Department of Community Health Sciences \& Hotchkiss Brain Institute. University of Calgary. Calgary, Canada.

** Department of Community Health Sciences, University of Calgary, Calgary, Canada. *** PhD Candidate, School of Public Health, University of Alberta, Edmonton, Canada

Address for correspondence:

Dr. Scott Patten

Professor, Department of Community Health Sciences

Faculty of Medicine

University of Calgary

3330 Hospital Drive NW

Calgary, AB, Canada

$\mathrm{T} 2 \mathrm{~N} 4 \mathrm{~N} 1$

Voice: 403-220-8752

FAX: 403-270-7307

Email: patten@ucalgary.ca

Index Words: Major depressive episode, Depressive disorders, Longitudinal studies, Obesity, Antidepressive Agents. 
Background: Cross-sectional studies have reported an association between episodes of major depression (MDE) and obesity. The objective of this longitudinal analysis was to determine whether MDE increases the risk of becoming obese over a 10 year period. Method: We used data from the Canadian National Population Health Survey (NPHS), a longitudinal study of a representative cohort of household residents in Canada. The incidence of obesity, defined as a body mass index (BMI) of $\geq 30 \mathrm{~kg} / \mathrm{m}^{2}$ was evaluated in respondents who were 18 years or older at the time of a baseline interview in 1994. MDE was assessed using a brief diagnostic instrument. Results: The risk of obesity was not elevated in association with MDE, either in unadjusted or covariate-adjusted analyses. The strongest predictor of obesity was a BMI in the overweight (but not obese) range. Effects were also seen for (younger) age, (female) sex, a sedentary activity pattern, low income and exposure to antidepressant medications. Unexpectedly, significant effects were seen for serotonin reuptake inhibiting antidepressants and venlafaxine, but not for tricyclic antidepressants nor antipsychotic medications. Conclusions: MDE does not appear to increase the risk of obesity. The cross-sectional associations that have been reported, albeit inconsistently, in the literature probably represent an effect of obesity on MDE risk. Pharmacologic treatment with antidepressants may be associated with an increased risk of obesity, and strategies to offset this risk may be useful in clinical practice.

Keywords: Obesity, Body Mass Index, Epidemiologic Studies, Longitudinal Studies, Depressive Disorder, Antidepressive Agents, Body Mass Index. 


\section{Introduction:}

In the US [1,2] and Canada [3] cross-sectional associations between obesity, defined in terms of body mass index (BMI), and major depressive episode (MDE) have been reported. This literature, however, has not been highly consistent. An analysis of data from the National Comorbidity Survey replication by Simon et al. found a statistically significant association between obesity (BMI $\geq 30 \mathrm{~kg} / \mathrm{m}^{2}$ ) and MDE, but the association was weak: OR = 1.2 for lifetime episodes and only 1.1 for past year MDE [1]. The association persisted after adjustment for age, sex and other psychiatric disorders. McIntyre et al. reported similar estimates [3]. In an analysis of a large scale epidemiologic data set, Carpenter et al. [4] reported a gender interaction in which a weak positive association was seen in women $(\mathrm{OR}=1.37)$ offset by a negative association in men $(\mathrm{OR}=0.63)$. McLaren et al. [5] also reported a significantly higher prevalence of mood disorders in obese compared to normal weight women but not men. On the other hand, a cross-sectional analysis of data from the National Health and Nutrition Examination Survey (NHANES) by Onyike et al. [2] found an association in both women $(\mathrm{OR}=1.8)$ and men $(\mathrm{OR}=1.7)$. However, the association in men did not attain statistical significance. Finally, in contrast to all of these results, a study from Finland [6] reported an association in men but not in women. The interpretation of these studies is limited by their cross-sectional study designs, which preclude estimation of risk and cannot clarify the temporal relationships between depression and obesity.

Cross-sectional associations may hypothetically be caused either by an effect of obesity on MDE incidence, an effect of MDE on obesity incidence, or effects of one condition on the persistence of the other. There is some evidence that obesity may 
increase the risk of MDE. In the Alameda County study of older adults (age 50 or more), Roberts et al. $[7,8]$ reported a doubling of the incidence of MDE in respondents who were obese at baseline. The Children in the Community Study found an increased risk in obese girls but not boys [9].

Another possibility is that MDE may increase the risk of developing obesity. This possibility has been relatively neglected in the literature and, again, the reported findings have been inconsistent. An analysis of prospective data from the Zurich cohort study reported an association between atypical depression and obesity, but the rate of increase in BMI over time was not significantly influenced by MDE [10]. The Zurich study used its own diagnostic interview, and its comparability to more commonly used interviews is questionable. Using data from the Children in the Community Study, Anderson et al. [11] reported that MDE in childhood predicted more rapid increases in BMI in females, but the reverse was seen in males. The Alameda County study of older adults did not find that MDE predicted subsequent obesity [12], nor did a population-based study of adolescents conducted in Dunedin [13].

In addition to the general lack of evidence concerning the incidence of obesity in people with MDE, a notable deficiency in this literature is a lack of control for psychotropic medication use. Only one epidemiologic study has included this variable, finding that it had no effect [14]. This study was conducted in 1981 and there have been large increases in the frequency and changes in the pattern of antidepressant use since then [15]. Tricyclic antidepressants, are generally thought to increase the risk of obesity, see review by Schwartz et al. [16]. The goal of this study was to determine whether MDE 
is associated with incident obesity in the general population of Canada, and to include adjustment for important covariates - including psychotropic medication use.

Method:

The NPHS is a longitudinal study based on a nationally representative community sample assembled by Statistics Canada (Canada's national statistical agency) in 1994/1995. Detailed information about the NPHS methods may be found on the Statistics Canada Web page (www.StatCan.ca). The longitudinal cohort included 17,276 participants, but the current analysis was restricted to $n=11,502$ respondents who were 18 years or older at the time of the initial 1994 interview and also non-obese (BMI less than $30 \mathrm{~kg} / \mathrm{m}^{2}$ ) at that time. Over the 10 year period, $26 \%$ of the original cohort was lost to follow-up. In addition, some respondents left the sampling frame because of institutionalization, death or homelessness. All of these respondents were included in the analysis, but were censored at the time of their last observation. The NPHS cohort has been interviewed every two years, such that five follow-up interviews are available covering the 1994 to 2004 time period. The NPHS interview includes self-report items assessing height and weight, making calculation of the BMI possible. The 1994 NPHS interviews were mostly conducted face to face, but the majority of follow-up interviews have been conducted over the telephone.

The NPHS interview included the Composite International Diagnostic Interview Short Form (CIDI-SF) [17] for MD, which assesses past year MDE. The CIDI-SF was administered by experienced and trained Statistics Canada interviewers. These 
interviewers, however, were not health professionals. The CIDI-SF is scored with a predictive probability algorithm based on the number of symptom-based criteria fulfilled during a 2-week period during the preceding year, and which also requires either depressed mood or loss of interest or pleasure, consistent with DSM-IV [18]. The instrument was scored at the $90 \%$ predictive cut-point in these analyses. This cut-off requires endorsement of 5 of 9 specified depressive symptoms during the same 2-week period, also consistent with DSM-IV criteria.

In the analysis, MDE was treated as a time varying factor, so that MDE status at the start of each 2-year incidence interval determined whether a respondent was in the exposed or non-exposed cohort during that interval. We modeled the effect of MDE on obesity incidence using a proportional hazards model. Because the NPHS employed interviews at discrete ( 2 year) time points, a proportional hazards model for grouped time data was used. This was fit as a generalized linear model of the binomial family with a complementary log-log link function. Jenkins has outlined procedures for implementation of these analyses in STATA [19]. The analyses presented here are nonparametric: time intervals are represented in the models using indicator variables with no assumption about the pattern of change in risk over time. The respondents developing obesity, dying or who were institutionalized were censored from the models at subsequent time points. Since obesity is a reversible outcome, a secondary analysis was conducted to examine the possible effect of MDE on persistence of obesity among those in the obese category at baseline $(\mathrm{n}=1867)$. After preliminary tabular and stratified analyses, crude (unadjusted) hazards ratios (HR) were calculated. Next, age and sex were added to the models. Age was depicted using dummy variables for five age groups: 18- 
$25,26-45,46-65$ and $66+$, with the latter category serving as a baseline group. In view of the literature (see above), age and sex by MDE interaction terms were assessed in all of the analyses.

The analysis included baseline overweight status (BMI $\geq 25 \mathrm{but}<30 \mathrm{~kg} / \mathrm{m}^{2}$ ) as a covariate. We considered this variable to be a potential confounder as it may plausibly be associated with obesity risk and with MDE. The NPHS defined an active (non-sedentary) lifestyle using a series of questions about recreational physical activity, metabolic demands of those activities and amount of time spent participating in them. Low income was defined as a household income of less than $\$ 30,000$ (Canadian). Low income may be related to obesity risk as a result of several mechanisms, including the low cost of highly palatable energy-dense foods [20]. The NPHS interviewers asked respondents to collect all of their medication containers and collected a record of medication use in the 2 days preceding the interview. These were coded using the World Health Organisation's Anatomic Therapeutic Classification (ATC). The ATC codes were used to identify the use of psychotropic medications including antipsychotic medications, sedative-hypnotics and various classes of antidepressant. All of the covariates were included in the models as time-varying characteristics and were selected for inclusion in the final models based on $\mathrm{p}<0.10$.

The NPHS used a multistage, stratified design that also included clustering to select eligible households. To correct for bias potentially resulting from this complex survey design, Statistics Canada recommends a bootstrap procedure that uses a set of 500 replicate weights. All of the estimates presented below are weighted in this way and the confidence intervals derive from the bootstrap procedure. All analyses were conducted at 
the Regional Research Data Centre on the University of Calgary campus, using STATA [21]. The study received approval from the University of Calgary Conjoint Ethics Board.

$\underline{\text { Results: }}$

There were 11,502 respondents eligible for inclusion in the analysis, see Figure 1. Of these, 5.6\% (95\% CI 5.1 - 6.2) had MDE at the baseline interview. Of the total sample, $3.8 \%$ (95\% CI $3.3-4.3)$ entered into the obese category in the initial 2-year follow-up period between 1994 and 1996 - the incidence being 3.5\% in women and $4.1 \%$ in men. In subsequent cycles, the incidence of obesity ranged from $2.9 \%$ to $4.0 \%$. Of those with MDE in 1994, 4.3\% (95\% CI 2.3 - 6.2) were obese in 1996 compared to $3.6 \%$ (95\% CI $3.1-4.1)$ of those without MDE.

Over the 10 year follow-up interval, the unadjusted HR for MDE was 1.1 (95\% CI $0.8-1.6)$. No interactions were identified when covariates and associated interaction terms were added to the model. Women had an elevated risk of obesity, as did those in the younger age categories, those without a physically active lifestyle, low income and those who were in the overweight BMI category. There was an association of obesity risk with use of serotonin-specific re-uptake inhibitors and venlafaxine, but no association for other antidepressant categories. However, inclusion of these covariates in a single model did not alter the (lack of) association between MDE and obesity incidence, see Table 1. 
Table 1. Proportional Hazards Model for Obesity, by MDE*

\begin{tabular}{llll}
\hline & HR & $95 \%$ CI & p-value \\
\hline MDE & 1.1 & $0.8-1.5$ & 0.70 \\
Female & 1.2 & $1.0-1.4$ & 0.03 \\
Age 18-25 & 3.2 & $2.3-4.3$ & $<0.001$ \\
Age 26-45 & 2.2 & $1.7-2.8$ & $<0.001$ \\
Age 46-65 & 1.8 & $1.4-2.3$ & $<0.001$ \\
Physically active lifestyle & 0.9 & $0.8-1.0$ & 0.02 \\
Low income & 1.3 & $1.2-1.5$ & $<0.001$ \\
Baseline BMI "overweight” & 12.5 & $10.2-15.3$ & $<0.001$ \\
Venlafaxine & 4.9 & $1.8-13.0$ & $<0.001$ \\
SRI & 1.9 & $1.2-3.2$ & 0.01 \\
\hline
\end{tabular}

* tricyclic antidepressants were not significantly associated with obesity risk: $\mathrm{HR}=1.3$, $95 \%$ CI $0.85-2.0, p=0.23$

Obesity is a reversible outcome. However, in the analyses presented above, respondents who developed obesity were subsequently removed from the population at risk. In order to determine the proportions moving from the obese to the non-obese categories we conducted a secondary analysis in which only those with a BMI $\geq 30 \mathrm{~kg} / \mathrm{m}^{2}$ at baseline were included. Of those with MDE at the 1994 baseline interview, $13.0 \%$ (95\% CI 5.1 - 20.8) moved into a non-obese BMI category by the next interview in 1996. However, a greater percentage of those in the non-MDE category made this transition, $22.4 \%$ (95\% CI 19.6 - 25.1). In the remaining cohort, the longer the respondents persisted in the obese category the less likely they were to become non-obese. Only $7.1 \%$ of those who were persistently obese between 1994 and 2002 became non-obese by 2004 . The crude HR for MDE suggested that those with MDE were less likely to move from obese to non-obese status ( $\mathrm{HR}=0.5,95 \%$ CI $0.3-1.0), \mathrm{p}=0.04$. However, after adjustment for other factors found to be related to recovery from obesity (age, sex, chronic conditions, low income) the effect of MDE became slightly weaker and no longer attained statistical significance, $\mathrm{HR}=0.6(95 \% \mathrm{CI} 0.3-1.1), \mathrm{p}=0.11$. 


\section{Discussion:}

These analyses uncovered no evidence that MDE increases the risk of obesity. In general, the analysis reproduced an expected pattern of obesity determinants, providing some degree of confidence about the results. The most notable finding is an apparent association between antidepressant use and obesity incidence. Notably, the association was seen for serotonin specific reuptake inhibitors and venlafaxine, which are generally believed to convey less risk of weight gain than other antidepressants. It is possible that this result may be due to confounding by prescriber expectancy. Physicians may specifically select these medications for use in patients they believe to be most at risk of weight gain. As such, those exposed to serotonin specific reuptake inhibitors and venlafaxine may be a group at higher risk of obesity for reasons other than their antidepressant medications. On the other hand, it is possible that these medications, which tend to reduce appetite in initial stages of treatment, may lead to weight gain with longer term use. An effect of this nature may represent a manifestation of tolerance to the effects of antidepressant medications [22]. Concern with the possibility that antidepressants may act as risk factors for obesity is magnified by recent large increases in the frequency of antidepressant treatment [23].

A limitation of the analysis is its reliance on self-reported height and weight, which could lead to an underestimation of obesity incidence [24]. The resulting misclassification bias might obscure an association between MDE and obesity incidence. Also, if both MDE and obesity were related to attrition, then bias may have been introduced because of loss of follow-up. The extent of bias is likely to be small, however, 
since MDE is not strongly associated with attrition from cohort studies [25,26]. Another limitation is misclassification that may have resulted from the timing of the interviews. The CIDI-SF identifies past year episodes, whereas the NPHS interviews were spaced two years apart. Some respondents with brief MDEs may not have been classified correctly in the analysis. If the resulting misclassification were non-differential (ie. the same in obese and non-obese respondents), the expected direction of bias would be towards the null. The CIDI-SF was not designed to identify atypical depressive episodes. The results, therefore, cannot be generalized to atypical forms of depression.

These results convey a cautionary message about the adverse health effects of depression. Reports of cross-sectional associations between obesity and MDE (although these have been inconsistent) might be interpreted as a basis for increasingly energetic antidepressant treatment. However, it appears that MDE itself is probably not a strong determinant of obesity. However, obesity may be associated with antidepressant treatment. Non-pharmacologic treatments for MDE such as cognitive behavioral therapy may be preferable for suitable individuals who are at risk of obesity. Notably, the protective effect of physical activity seen in Table 1 may be a valuable ancillary result, as behavioral activation strategies are often beneficial in depression management. Participation in extra physical activity may also have the added benefit of protecting against antidepressant induced weight gain. 
Acknowledgments and Disclaimer: Dr. Patten is a Health Scholar with the Alberta Heritage Foundation for Medical Research (www.ahfmr.ab.ca). This analysis was based on data collected by Statistics Canada. The analysis and interpretations presented do not reflect those of Statistics Canada. Dr. McLaren is a Population Health Investigator with the Alberta Heritage Foundation for Medical Research. Dr. Eliasziw is a Senior Scholar with the Alberta Heritage Foundation for Medical Research. Ms. Brown is supported by a Fellowship from the Canadian Institutes for Health Research. This work was supported by a grant from the Canadian Institutes for Health Research. None of the authors have conflicts of interest related to this research. 


\section{Reference List}

[1] Simon GE, Von Korff M, Saunders K, Miglioretti DL, Crane PK, van Belle G, Kessler RC: Association between obesity and psychiatric disorders in the US adult population. Arch Gen Psychiatry 2006;63:824-830.

[2] Onyike CU, Crum RM, Hochang BL, Lyketsos CG, Eaton WW: Is obesity associated with major depression? Results from the third National Health and Nutrition Examination Survey. Am J Epidemiol 2003;158:1139-1147.

[3] McIntyre RS, Konarksi JZ, Wilkins K, Soczynska JK, Kennedy SH: Obesity in bipolar disorder and major depressive disorder: Results from a national community health survey on mental health and well-being. Can J Psychiatry 2006;51:274-280.

[4] Carpenter KM, Hasin DS, Allison DB, Faith MS: Relationships between obesity and DSM-IV major depressive disorder, suicide ideation, and suicide attempts: results from a general population study. Am J Publ Hlth 2000;90:251-257.

[5] McLaren L, Beck CA, Patten SB, Fick GH, Adair CE: The relationship between body mass index and mental health. A population-based study of the effects of the definition of mental health. Soc Psychiatry Psychiatr Epidemiol 2008;43:63-71.

[6] Lindeman S, Hämäläinen J, Isometsä E, Kaprio J, Poikolainen K, Heikkinen M, Aro H: The 12-month prevalence and risk factors for major depressive episode in Finland: representative sample of 5993 adults. Acta Psychiatr Scand 2000;102:178-184.

[7] Roberts RE, Strawbridge WJ, Deleger S, Kaplan GA: Are the fat more jolly? Ann Behav Med 2002;24:169-180.

[8] Roberts RE, Deleger S, Strawbridge WJ, Kaplan GA: Prospective association between obesity and depression: evidence from the Alameda County Study. In J Obesity 2003;27:514-521.

[9] Anderson SE, Cohen P, Naumova EN, Jacques PF, Must A: Adolescent obesity and risk for subsequent Major Depressive Disorder and anxiety disorder: prospective evidence. Psychosom Med 2007;69:740-747.

[10] Hasler G, Pine DS, Gamma A, Milos G, Ajdacic V, Eich D, Rössler W, Angst J: The associations between psychopathology and being overweight: a 20-year prospective study. Psychol Med 2004;34:1047-1057.

[11] Anderson SE, Cohen P, Naumova EN, Must A: Association of depression and anxiety disorders with weight change in a prospective community-based study of children followed up into adulthood. Arch Pediatr Adolesc Med 2006;160:285291. 
[12] Domalanta DD, Risser WL, Roberts RE, Risser JM: Prevalence of depression and other psychiatric disorders among incarcerated youths. J Am Acad Child Adolesc Psychiatry 2003;42:477-484.

[13] Bardone AM, Moffitt TE, Caspi A, Dickson N, Stanton WR, Silva PA: Adult physical health outcomes of adolescent girls with conduct disorder, depression, and anxiety. J Am Acad Child Adolesc Psychiatry 1998;37:594-601.

[14] Noppa H, Hällstrtöm T: Weight gain in adulthood in relation to socioeconomic factors, mental illness and personality traits: A prospective study of middle-aged women. J Psychosom Res 1981;25:83-89.

[15] Beck CA, Patten SB, Williams JVA, Wang JL, Currie SR, Maxwell CJ, elGuebaly N: Antidepressant utilization in Canada. Soc Psychiatry Psychiatr Epidemiol 2005;40:799-807.

[16] Schwartz TL, Nihalani N, Jindal S, Virk S, Jones N: Psychiatric medicationinduced obesity: a review. Obes Rev 2004;5:115-121.

[17] Kessler RC, Andrews G, Mroczek D, Ustun B, Wittchen HU: The World Health Organization Composite International Diagnostic Interview Short-Form (CIDISF). Int J Methods Psychiatr Res 1998;7:171-185.

[18] American Psychiatric Association: Diagnostic and Statistical Manual of Mental Disorders (DSM-IV-TR). Washington, American Psychiatric Association, 2000.

[19] Jenkins SP: Discrete time proportional hazards regression. STATA Technical Bulletin 1997;STB-39:22-31.

[20] Drewnowski A, Specter SE: Poverty and obesity: the role of energy density and energy costs. Am J Clin Nutr 2004;79:6-16.

[21] Stata Corporation. Stata. [9.0]. 2005. College Station, TX, Stata Corporation. Ref Type: Computer Program

[22] Fava GA, Tomba E, Grandi S: The road to recovery from depression--don't drive today with yesterday's map. Psychother Psychosom 2007;76:260-265.

[23] Mojtabai R: Increase in antidepressant medication in the US adult population between 1990 and 2003. Psychother Psychosom 2008;77:83-92.

[24] Tjepkema M: Adult obesity. Health Rep 2006;17:9-25.

[25] de Graaf R, Bijl RV, Smit F, Ravelli A, Vollebergh WA: Psychiatric and sociodemographic predictors of attrition in a longitudinal study. Am J Epidemiol 2000;152:1047. 
[26] Badawi MA, Eaton WW, Myllyluoma J, Weimer LG, Gallo J: Psychopathology and attrition in the Baltimore ECA 15-year follow-up 1981-1996. Soc Psychiatry Psychiatr Epidemiol 1999;34:91-98. 